УДК 621.642.07

\author{
ОЦЕНКА РАБОТОСПОСОБНОСТИ ШТУЦЕРНЫХ УЗЛОВ \\ СОСУДОВ ДАВЛЕНИЯ В УСЛОВИЯХ ЦИКЛИЧЕСКОГО РЕЖИМА \\ ЭКСПЛУАТАЦИИ
}

\title{
PERFORMANCE ESTIMATION OF THE PRESSURE VESSELS UNITS IN THE CONDITIONS OF THE CYCLIC OPERATION MODE
}

\author{
А.М. Файрушин, А.В. Исламова \\ Уфимский государственный нефтяной технический университет, \\ Уфа, Российская Федерация \\ Airat M. Fairushin, Alena V. Islamova \\ Ufa State Petroleum Technological University, Ufa, Russian Federation \\ e-mail: otsk@ rusoil.net
}

Аннотация. Одними из ключевых и наиболее использующимся вариантом укрепления отверстий в различных сосудах и аппаратах, которые используются в технологических процессах на нефтехимических и нефтеперерабатывающих предприятиях, являются внедрение и использование накладного кольца. Используемая на сегодняшний день технология сборки и сварки накладных колец, совместно с самой конструкцией, является достаточно проработанной, с богатым опытом эксплуатации на современных предприятиях. Несмотря на это сегодня существуют вопросы, касающиеся ее изготовления, которые требуют более углубленного исследования.

Одним из основных методов решения существующих проблем контроля качества сборки является контроль сборки узла укрепления отверстия. Необходимо отметить, что требования, изложенные в ГОСТ Р 52630-2006, об ограничении размера зазора до 3 мм на практике не всегда выполняется. 
Основной задачей представленного исследования является разработка лабораторного стенда, предназначенного для исследования ресурса работы узла приварки штуцера. В данной работе представлена возможная конструкция данной лабораторной установки, также предоставлены результаты проведенного практического исследования четырех вариаций исполнения укрепления узла врезки.

Abstract. One of the key and most used option for strengthening holes in various vessels and apparatus that are used in technological processes at petrochemical and oil refineries is the introduction and use of a reinforcing (overhead) ring. The technology used today for the assembly and welding of reinforcing rings, together with the structure itself, are sufficiently developed with extensive operating experience in modern enterprises. Despite this, today there are questions regarding its manufacture that require more in-depth research. One of the main methods for solving the existing problems of assembly quality control is the control of the assembly of the hole reinforcement assembly. It should be noted that the requirements set out in GOST R 52630-2006 to limit the size of the gap to $3 \mathrm{~mm}$ are not always met in practice.

One of the main tasks of the presented study is the development of a laboratory stand designed to study the service life of the choke welding unit. This paper presents a possible design of this laboratory setup, also provides the results of a practical study of four variations in the execution of reinforcement of the tie-in unit.

Ключевые слова: узел ответвления; сосуды давления; накладное кольцо; поликарбамид; врезка; тройниковое соединение

Key words: branch node; pressure vessel; ring; polyuria; insert; tee connection 
На сегодняшний день промышленная безопасность имеет колоссальное значение ввиду повышения статистики травматизма на предприятиях и в промышленности. Одними из основных элементов технологических систем химических предприятий и смежных отраслей являются сосуды и аппараты, работающие под давлением. Актуальность промышленной безопасности в этом отношении связана с безопасной эксплуатацией сосудов с целью обеспечения эффективного функционирования и развития современных промышленных предприятий. Продолжая вопрос изучения актуальности представленной темы, необходимо отметить, что актуальность подобных задач определяется экономическими причинами, в частности высокой стоимостью оборудования, необходимостью использования устаревающего оборудования, уже отработавшего свой жизненный цикл, а также конкуренцией в условиях рыночной экономики, требующей снижение себестоимости производства. Именно в совокупности данных факторов актуальность разработки лабораторных стендов для оценки ресурса работы сосудов, работающих под давлением и гарантирующих безопасную эксплуатацию, имеет колоссальный характер на сегодняшний день [1].

Обеспечение безопасной и безаварийной работы производства, повышение надёжности оборудования и технологических процессов составляют основные задачи нефтеперерабатывающих заводов и нефтегазохимических производств.

Сварные сосуды и аппараты, работающие под давлением, являются основными представителями различных технологических установок нефтеперерабатывающих предприятий. Безаварийная эксплуатация данного оборудования обеспечивает безопасное функционирование и развитие современных предприятий. Как показывает опыт эксплуатации, на сегодняшний день остаются нерешёнными вопросы в части обеспечения качества изготовления и мониторинга данного оборудования, особенно в трудно контролируемых узлах аппаратов $[2,3]$. 
К одним из таких мест относится узел приварки штуцера к корпусу аппарата (штуцерный узел), а именно исполнение данного узла с укреплением отверстия накладным кольцом. Данный узел в различных исполнениях присутствует в каждом аппарате и является одним из потенциально опасных мест ввиду его конструктивных особенностей.

До 30-40 \% разрушений в аппаратах приходится на штуцерные узлы. Это объясняется не только тем, что в этих узлах ввиду разной деформационной способности сварные швы деталей испытывают воздействие объемно-напряженного состояния, но и тем, что в данных узлах обнаруживается большое количество технологических дефектов, возникающих ещё в процессе изготовления аппарата, поэтому такие узлы являются наиболее ответственными с точки зрения обеспечения надёжности конструкции в целом [4].

Существующая на сегодняшний день технология сборки с приваркой укрепляющих колец - это достаточно проработанная технология, имеющая большой опыт использования. Несмотря на это, на современных предприятиях далеко не всегда достигается допускаемый зазор относительно обечайки и накладной конструкции в 3 мм Данный фактор напрямую влияет на работоспособность данного узла. Практически всегда снижение зазора по периметру кольца производится посредством ударного инструмента, что также является негативным фактором производства, причем в частных случаях - это априори недопустимо во время создания сосудов и аппаратов [5].

Данные факторы определяют актуальность вопросов модернизации узла приварки штуцера, а также создания исследовательской установки с целью определения работоспособности штуцерных узлов, имеющих различного рода конструктивные исполнения.

В процессе эксплуатации на узел штуцера воздействует не только внутреннее давление в аппарате, но и нагрузка от присоединённого трубопровода, установленной трубопроводной арматуры и т.д. [6]. 
Для оценки влияния осевой нагрузки на напряжённое состояние узлов приварки штуцера к корпусу аппарата была разработана модель лабораторного стенда (рисунок 1).

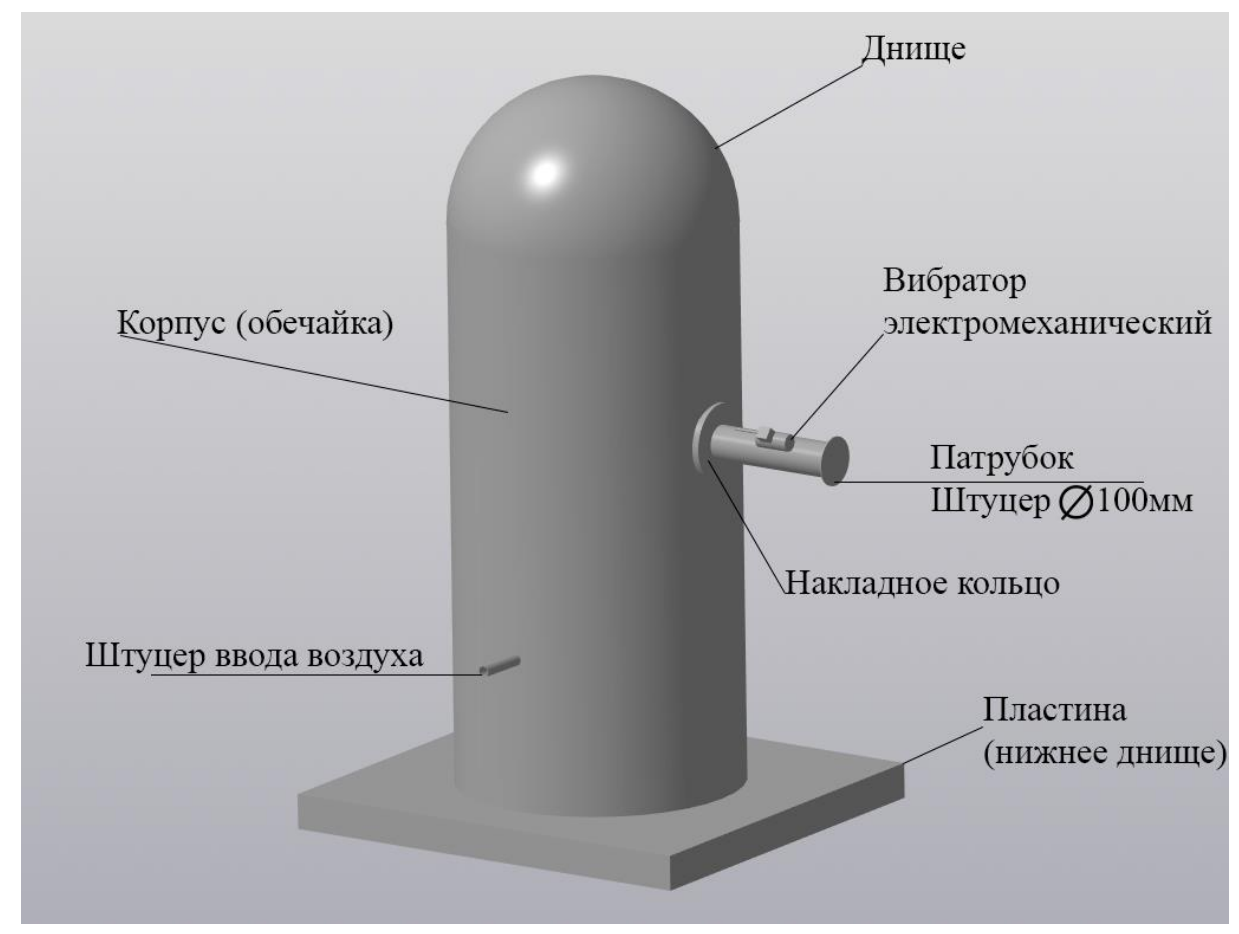

Рисунок 1. Модель лабораторного стенда

Установка представляет собой вертикальный цилиндрический аппарат, с двух сторон заглушенный эллиптическими крышками. В нижней части корпуса аппарата установлен штуцер для подачи воздуха (создания внутреннего давления). На обечайке установлен экспериментальный заглушенный с торца патрубок, имитирующий «рабочий» штуцер, на который устанавливается вибратор для создания циклической нагрузки.

Практические исследования работоспособности различных накладных конструкций укрепления штуцерного узла производились на изготовленном в натуральную величину экспериментальном аппарате в соответствии с рисунком 1. Обечайка имеет высоту 1,6 м; диаметр 0,8 м; толщину стенки 0,008 м; изготовлена из стали 09Г2С.

На рисунке 2 представлено фото данной экспериментальной установки. 

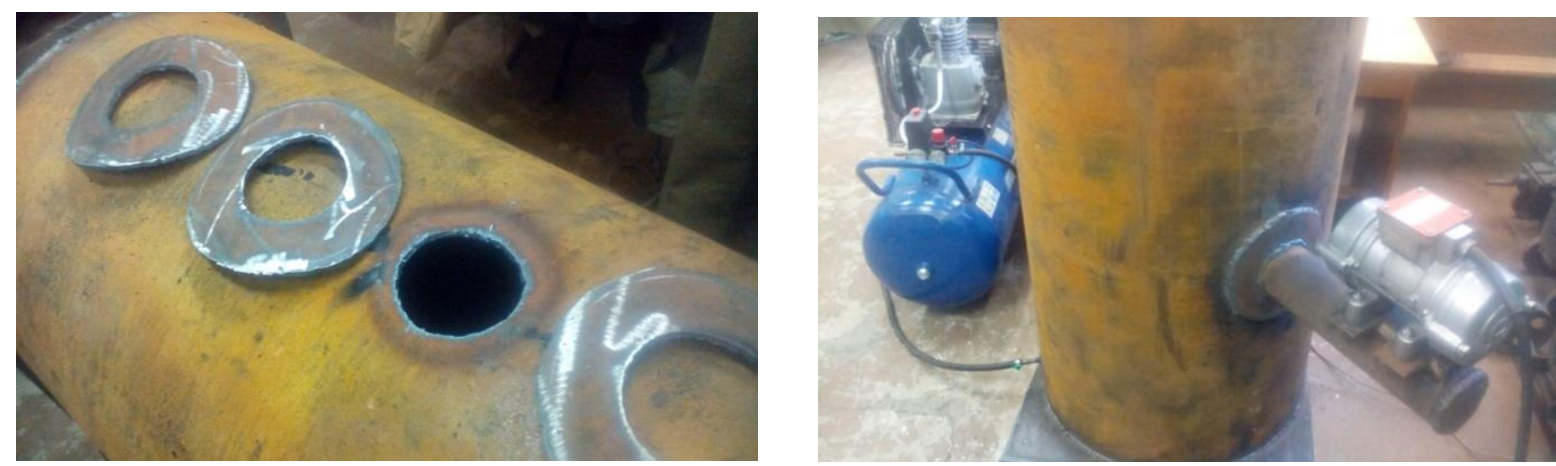

Рисунок 2. Фото экспериментальной установки

В качестве нагрузки параллельно с созданием внутреннего давления производили циклические нагрузки на штуцер. На краю штуцера был установлен электромеханический вибратор марки ИВ, создающий колебания с частотой вибрации 50 Гц и обеспечивающий нагрузку 1 кН.

Схема нагружения образцов приведена на рисунке 3.

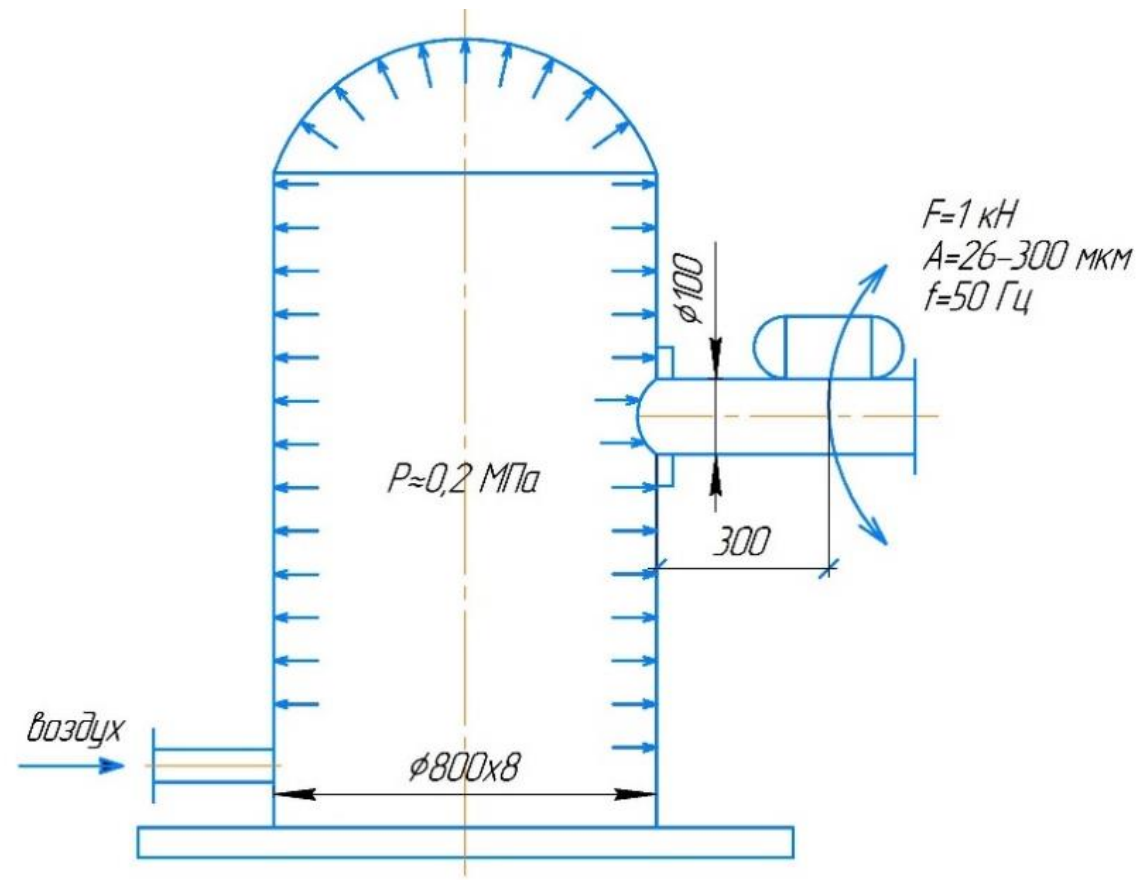

Рисунок 3. Схема нагружения образцов

Описываемый эксперимент проводился в лабораторных условиях. Температура воздуха составляла $18-20{ }^{\circ} \mathrm{C}$. Анализ колебаний вибрации производился посредством измерительного прибора Вибротест МГ-4. Полученные результаты данного эксперимента показали, что частота 
вибрации находится на уровне 45,4-49,6 Гц. Амплитуда колебаний вблизи штуцерного узла составляла 260-300 мкм.

Во внутреннее пространство сосуда с помощью компрессора подавался воздух, при этом давление в сосуде во время эксперимента имело статистическое значение и было равно 2 атм (0,2 МПа).

Ресурс работы аппарата оценивался относительно времени его герметичности при воздействии нагрузки. Герметичность определяли посредством визуального обнаружения течения воздуха в сварных соединениях штуцерного узла при использовании мыльного раствора.

Испытания на работоспособность при данных нагрузках производились для четырёх исполнений образцов:

- с укреплением одним накладным кольцом;

- с послойным укреплением накладными кольцами;

- без укрепления;

- с укреплением конструкцией из поликарбамида.

Для определения количество циклов время работы до обнаружения течи умножалась на количество циклов в минуту, воспроизводимых вибрационным устройством. Измерения проводились секундомером с точностью до 1 с. В результате испытаний получены следующие значения (таблица 1).

На гистограмме, приведённой на рисунке 4, наглядно показаны результаты испытания образцов на работоспособность до появления течи с различными конструктивными исполнениями.

Результаты эксперимента показали, что ресурс работы штуцерного узла без укрепления накладным кольцом значительно снижается - на 26 \%, а с укреплением накладной конструкцией из поликарбамида - на 6 \%. 
Таблица 1. Результаты испытания

\begin{tabular}{|c|c|c|c|c|}
\hline \multirow{2}{*}{$\begin{array}{c}\text { Номер } \\
\text { образца }\end{array}$} & $\begin{array}{c}\text { С } \\
\text { укрепляющим } \\
\text { кольцом }\end{array}$ & $\begin{array}{c}\text { С послойным } \\
\text { укреплением } \\
\text { накладными } \\
\text { кольцами }\end{array}$ & $\begin{array}{c}\text { Без } \\
\text { укрепляющего } \\
\text { кольца }\end{array}$ & $\begin{array}{c}\text { С укреплением } \\
\text { накладной } \\
\text { конструкции из } \\
\text { поликарбамида }\end{array}$ \\
\hline 1 & 755220 & 828060 & 557340 & 703920 \\
\hline 2 & 749040 & 829980 & 543360 & 707880 \\
\hline 3 & 752820 & 811860 & 559980 & 712500 \\
\hline $\begin{array}{c}\text { Среднее } \\
\text { значение }\end{array}$ & 752360 & 823300 & 553560 & 708100 \\
\hline
\end{tabular}

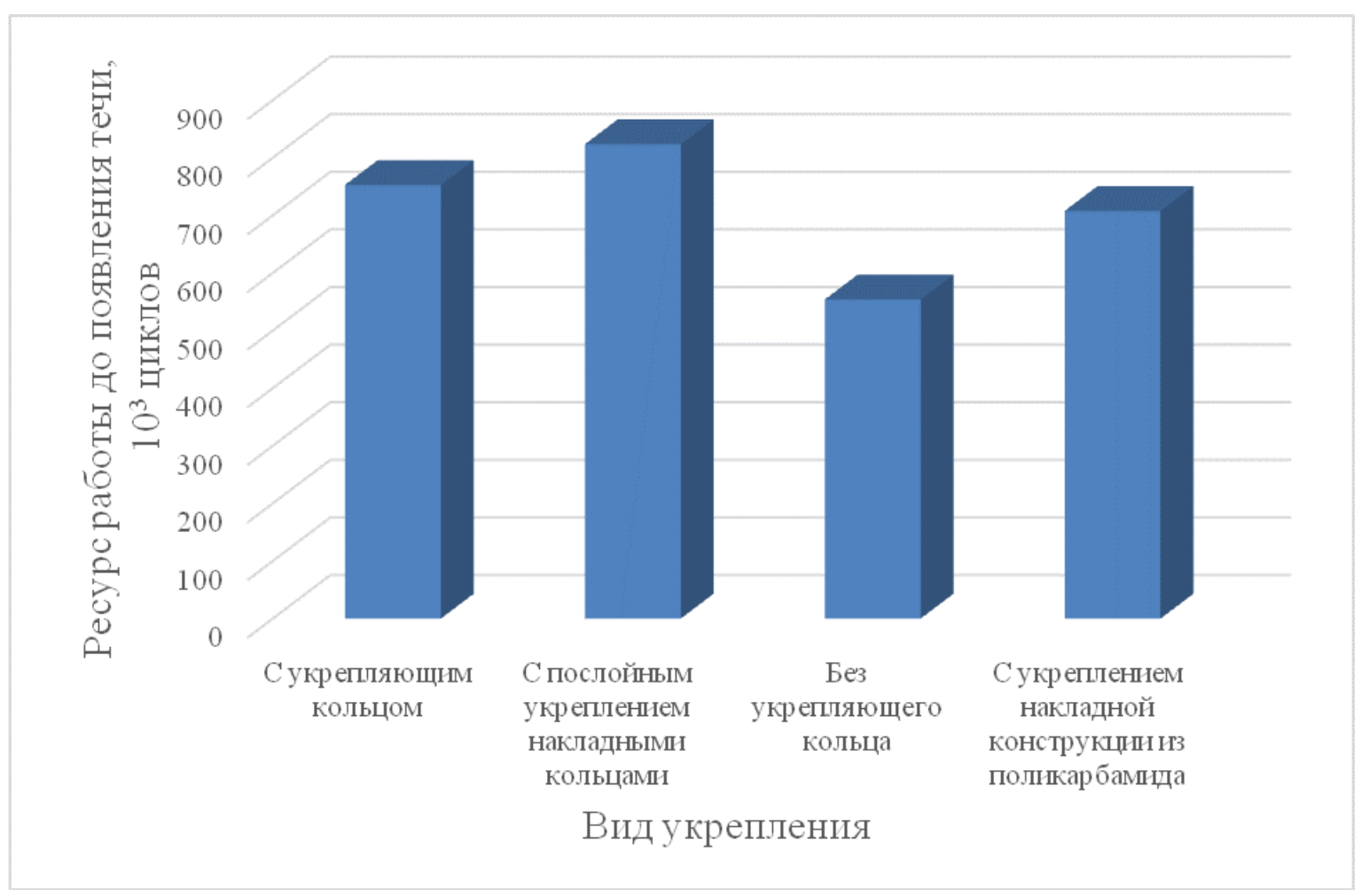

Рисунок 4. Результаты испытания образцов с различными конструктивными исполнениями на работоспособность до появления течи

При применении конструкции с послойным укреплением ресурс работы узла при таком нагружении увеличивается на $9 \%$. 


\section{Выводы}

1. Тройниковые соединения подвержены значительным нагрузкам, вследствие чего требуют особое внимание к качеству изготовления и минимизации негативного воздействия на тело аппарата от сварки.

2. Повышение безопасности участка ответвления и врезки достигается путём установки плоской усиливающей накладки на трубопроводе-отводе. На основании проведенных испытаний можно предположить, что применение конструкции с послойным укреплением позволит уменьшить напряжения и увеличить срок службы данного узла на $9 \%$ по сравнению с существующими технологиями установки накладных колец.

3. Результаты исследования показали, что узел врезки также можно укреплять конструкцией из поликарбамида. Данные конструкции незначительно уступают в работоспособности штуцерным узлам с накладными кольцами, но позволяют избавиться от дефектов, получаемых при проведении сварочно-монтажных работ в процессе установки накладных колец.

\section{Список используемых источников}

1. ГОСТ Р 52630-2006. Сосуды и аппараты стальные сварные. Общие технические условия. М.: Стандартинформ, 2006. 68 с.

2. Сабитов M.X., Поникаров С.И. Анализ напряжённодеформированного состояния штуцерных узлов в расчёте на малоцикловую прочность сосудов давления // Вестник Казанского технологического университета. 2011. № 20. С. 208-212.

3. Гумеров А.К., Шмаков А.К., Хайрутдинов Ф.Ш. Механизмы разрушения магистральных трубопроводов с приварными элементами // Электронный научный журнал «Нефтегазовое дело». 2007. № 1. C. 90. URL: http://ogbus.ru/files/ogbus/authors/GumerovAK/GumerovAK_1.pdf (дата обращения: 11.01.2021). 
4. Аписов И.В., Четверткова О.В., Каретников Д.В., Ибрагимов И.Г., Закиров Т.Э. Анализ напряжённого состояния укреплённого накладным кольцом штуцерного узла с учетом дефектов сборки // Электронный научный журнал «Нефтегазовое дело». 2014. № 5. C. 223-237. URL: http://ogbus.ru/files/ogbus/issues/5_2014/ogbus_5_2014_p223237_ApisovIV_ru.pdf (дата обращения: 12.01.2021).

5. Зарипов М.З., Файрушин А.М., Яковлева Д.Н. Влияние качества сборки узла приварки штуцера на ресурс работы корпуса аппарата // Проблемы сбора, подготовки и транспорта нефти и нефтепродуктов. 2018. Вып. 3 (113). C. 143-150. DOI: 10.17122/ntj-oil-2018-3-143-150.

6. Яковлева Д.Н., Файрушин А.М., Исламова А.В. Повышение безопасности при эксплуатации узлов ответвления трубопроводов с помощью укрепляющих (накладных) колец // Сетевое издание «Нефтегазовое дело». 2018. № 6. С. 43-59. URL: http://ogbus.ru/files/ogbus/issues/6_2018/ogbus_6_2018_p43-59.pdf （дата обращения: 12.01.2021). DOI: 10.17122/ogbus-2018-6-43-59.

\section{References}

1. GOST $R$ 52630-2006. Sosudy i apparaty stal'nye svarnye. Obshchie tekhnicheskie usloviya [State Standard R 52630-2006. Steel Welded Vessels and Apparatus. General Specifications]. Moscow, Standartinform Publ., 2006. 68 p. [in Russian].

2. Sabitov M.Kh., Ponikarov S.I. Analiz napryazhenno-deformirovannogo sostoyaniya shtutsernykh uzlov $\mathrm{v}$ raschete na malotsiklovuyu prochnost' sosudov davleniya [Analysis of the Stress-Strain State of Choke Assemblies Based on the Low-Cycle Strength of Pressure Vessels]. Vestnik Kazanskogo tekhnologicheskogo universiteta - Herald of Kazan Technological University, 2011, No. 20, pp. 208-212. [in Russian]. 
3. Gumerov A.K., Shmakov A.K., Khairutdinov F.Sh. Mekhanizmy razrusheniya magistral'nykh truboprovodov $\mathrm{s}$ privarnymi elementami [Destruction Mechanisms of Main Pipelines with Welded Elements]. Elektronnyi nauchnyi zhurnal «Neftegazovoe delo»-Electronic Scientific Journal «Oil and Gas Business», 2007, No. 1, pp. 90. URL: http://ogbus.ru/files/ogbus/authors/GumerovAK/GumerovAK_1.pdf (accessed 11.01.2021). [in Russian].

4. Apisov I.V., Chetvertkova O.V., Karetnikov D.V., Ibragimov I.G., Zakirov T.E. Analiz napryazhennogo sostoyaniya ukreplennogo nakladnym kol'tsom shtutsernogo uzla s uchetom defektov sborki [Deflected Mode's Analyze of Vessel's Connecting Pipe Strengthened with Restorative Ring]. Elektronnyi nauchnyi zhurnal «Neftegazovoe delo» - Electronic Scientific Journal «Oil and Gas Business», 2014, No. 5, pp. 223-237. URL: http://ogbus.ru/files/ogbus/issues/5_2014/ogbus_5_2014_p223237_ApisovIV_ru.pdf (accessed 12.01.2021). [in Russian].

5. Zaripov M.Z., Fairushin A.M., Yakovleva D.N. Vliyanie kachestva sborki uzla privarki shtutsera na resurs raboty korpusa apparata [Influence of Welded Assembly Quality on the Apparatus Casing Operation Resource]. Problemy sbora, podgotovki i transporta nefti i nefteproduktov - Problems of Gathering, Treatment and Transportation of Oil and Oil Products, 2018, Issue 3 (113), pp. 143-150. DOI: 10.17122/ntj-oil-2018-3-143-150. [in Russian].

6. Yakovleva D.N., Fairushin A.M., Islamova A.V. Povyshenie bezopasnosti pri ekspluatatsii uzlov otvetvleniya truboprovodov $\mathrm{s}$ pomoshch'yu ukreplyayushchikh (nakladnykh) kolets [Improving Safety During Operating Pipelets Answering Units by Strengthening (Folding) Rings]. Setevoe izdanie «Neftegazovoe delo»-Online Edition «Oil and Gas Business», 2018, No. 6, pp. 43-59. URL: http://ogbus.ru/files/ogbus/issues/6_2018/ogbus_6_2018_p4359.pdf (accessed 12.01.2021). DOI: 10.17122/ogbus-2018-6-43-59. [in Russian]. 


\section{Сведения об авторах}

\section{About the authors}

Файрушин Айрат Миннуллович, канд. техн. наук, доцент, заведующий кафедрой «Оборудование и технологии сварки и контроля», УГНТУ, г. Уфа, Российская Федерация

Airat M. Fairushin, Candidate of Engineering Sciences, Associate Professor, Head of Equipment and Technologies for Welding and Control Department, USPTU, Ufa, Russian Federation

e-mail: otsk@ rusoil.net

Исламова Алена Вячеславовна, магистрант кафедры «Оборудование и технологии сварки и контроля», УГНТУ, г. Уфа, Российская Федерация

Alena V. Islamova, Undergraduate Student of Equipment and Technologies for Welding and Control Department, USPTU, Ufa, Russian Federation

e-mail: alena.islamova.1997@mail.ru 